\title{
Treatment of backscattering in a gas of interacting fermions confined to a one-dimensional harmonic atom trap
}

\author{
Gao Xianlong, F. Gleisberg, F. Lochmann, and W. Wonneberger \\ Abteilung für Mathematische Physik, Universität Ulm, D89069 Ulm, Germany
}

(Dated: November 1, 2018)

\begin{abstract}
An asymptotically exact many body theory for spin polarized interacting fermions in a onedimensional harmonic atom trap is developed using the bosonization method and including backward scattering. In contrast to the Luttinger model, backscattering in the trap generates one-particle potentials which must be diagonalized simultaneously with the two-body interactions. Inclusion of backscattering becomes necessary because backscattering is the dominant interaction process between confined identical one-dimensional fermions. The bosonization method is applied to the calculation of one-particle matrix elements at zero temperature. A detailed discussion of the validity of the results from bosonization is given, including a comparison with direct numerical diagonalization in fermionic Hilbert space. A model for the interaction coefficients is developed along the lines of the Luttinger model with only one coupling constant $K$. With these results, particle densities, the Wigner function, and the central pair correlation function are calculated and displayed for large fermion numbers. It is shown how interactions modify these quantities. The anomalous dimension of the pair correlation function in the center of the trap is also discussed and found to be in accord with the Luttinger model.
\end{abstract}

PACS numbers: PACS numbers: 71.10.Pm, 05.30.Fk, 03.75.Fi

\section{INTRODUCTION}

The achievement of Bose-Einstein condensation in dilute ultracold gases [1] stimulated the theoretical interest in trapped fermionic many body systems [2, 3, 4], especially their superfluid properties $5,6,6,7,8]$. Recent experimental successes in obtaining degeneracy in three-dimensional Fermi vapors [9, 10, 11, 12, 13] intensified the interest in confined Fermi gases.

Using microtrap technology 14, 15, 16, 17, 18, it is conceivable to realize a neutral ultracold quantum gas of trapped quasi one-dimensional degenerate fermions.

In many cases, identical spin polarized fermions experience only a weak residual interaction because s-wave scattering is forbidden. This makes the question of interactions in a one-component spin polarized fermion system somewhat academic. Possible exceptions are Feshbach resonance enhanced scattering between atoms [19] and electric dipoledipole interactions in the case of polar molecules [20].

The confinement of a trapped ultracold gas can be realized by a harmonic potential. We have developed an asymptotically exact theory of interacting one-dimensional fermions confined to a harmonic trap. It is based on the bosonization method known from the theory of Luttinger liquids (cf. 21, 22, 23, 24|) and exploits the linearity of the energy spectrum of free oscillator states. The method was presented in 25] for the one-component gas with forward scattering and extended in [26] to the case of two components. This model must be seen as a soft boundary alternative to the well studied case of one-dimensional interacting fermions confined by hard walls [27, 28, 29, 30, 31, 32, 33.

Meanwhile, an investigation of the interaction matrix elements for one-dimensional identical fermions in the harmonic trap revealed [34] that backscattering dominates forward scattering unless the pair potential is long ranged. Unlike in the case of the Luttinger model, backscattering cannot be taken into account by merely renormalizing the forward coupling constants. This is due to the one-branch structure of the present model, which generates one-particle potentials from the backward scattering when the latter is brought into the form of an effective forward scattering. These one-particle potentials must be diagonalized simultaneously with the two particle interactions from forward scattering. We solve this problem by supplementing the squeezing transformation with an appropriate displacement transformation.

The paper is organized as follows: Section II describes the model and classifies the scattering processes for fermions in the one-dimensional harmonic trap. Section III gives the solution of the backscattering problem for the one-particle matrix elements using the bosonization method. Section IV discusses the validity of the bosonization scheme and compares the results with those from direct numerical diagonalization in fermionic Hilbert space. Sec. V presents results for several quantities of interest: Occupation probabilities, particle and momentum densities, and the central pair correlation function, which are all derivable from the one-particle matrix elements. We employ a model of the interaction coefficients developed in analogy with the Luttinger model. It is characterized by just one coupling constant $K$ and by a small decay parameter $r \ll 1$. This approach is described in the Appendix. 


\section{THEORY}

\section{A. Description of the model}

We consider spin polarized fermionic atoms interacting via the pair interaction operator

$$
\hat{V}=\frac{1}{2} \sum_{m n p q} V(m, p ; q, n)\left(\hat{c}_{m}^{\dagger} \hat{c}_{q}\right)\left(\hat{c}_{p}^{\dagger} \hat{c}_{n}\right)
$$

The fermions are confined to a highly anisotropic axially symmetric harmonic trap. The trap potential is

$$
V(x, y, z)=\frac{1}{2} m_{A} \omega_{\ell}^{2} z^{2}+\frac{1}{2} m_{A} \omega_{\perp}^{2}\left(x^{2}+y^{2}\right) \equiv V_{z}(z)+V_{\rho}(\rho) .
$$

The atom mass is denoted $m_{A}$ and $z$ is the one-dimensional coordinate in the elongated axial direction of the trap. The trap frequencies are $\omega_{\ell}$ and $\omega_{\perp} \gg \omega_{\ell}$. The quasi one-dimensional Fermi gas is characterized by $N\left(\leq \omega_{\perp} / \omega_{\ell}\right)$ identical fermions filling the first $N$ one-particle levels

$$
\hbar \omega_{n}=\hbar \omega_{\ell}(n+1 / 2), \quad n=0,1, \ldots
$$

of the one-dimensional harmonic potential $V_{z}$, while the transverse part of each unperturbed wave function remains the transverse ground state $\psi_{\perp 0}(x) \psi_{\perp 0}(y)$.

The unperturbed one-dimensional Fermi energy is

$$
\epsilon_{F}=\hbar \omega_{\ell}(N-1)+\frac{1}{2} \hbar \omega_{\ell}=\hbar \omega_{\ell}\left(N-\frac{1}{2}\right) .
$$

The Fermi energy is only slightly smaller than the excitation energy $\hbar \omega_{\perp}$ of the first excited transverse state in the case of $N=N_{\max }=$ largest integer in $\omega_{\perp} / \omega_{\ell}$. We have in mind a situation when the filling factor $F \equiv N / N_{\max }$ is small enough so that the Fermi level is well below this excitation energy. The above assumption about the transverse wave function is then justified. Furthermore, there still exists a macroscopic number of possible excitations of the one-dimensional Fermi sea that do not violate this condition.

The operators in $\hat{c}_{m}^{\dagger}$ and $\hat{c}_{q}$ in Eq. (1) denote fermion creation and destruction operators, respectively. They obey the fermionic algebra $\hat{c}_{m} \hat{c}_{n}^{\dagger}+\hat{c}_{n}^{\dagger} \hat{c}_{m}=\hat{\delta}_{m, n}$. This ensures that each oscillator state with single particle wave function

$$
\psi_{n}(z)=\sqrt{\frac{\alpha}{2^{n} n ! \pi^{1 / 2}}} e^{-\alpha^{2} z^{2} / 2} H_{n}(\alpha z)
$$

and energy $\epsilon_{n}=\hbar \omega_{n}$ is at most singly occupied. The intrinsic length scale of the system is the oscillator length $l=\alpha^{-1}$ where $\alpha$ is defined by $\alpha^{2}=m_{A} \omega_{\ell} / \hbar$. $H_{n}$ denotes a Hermite polynomial. The non-interacting Hamiltonian $\hat{H}_{0}=\hbar \sum_{n=0}^{\infty} \omega_{n} \hat{c}_{n}^{+} \hat{c}_{n}$ has linear dispersion and incorporates exactly the harmonic trapping potential.

The interaction matrix elements $V(m, p ; q, n)$ in Eq. (11) are calculated from effective one-dimensional pair potentials using the harmonic oscillator states (15). Thus each individual interaction matrix element contains information about the harmonic trap.

In a degenerate Fermi system, the most relevant states are those near the Fermi energy, i.e. $m \approx p \approx q \approx n \approx N$. This limited number of interaction matrix elements can be further reduced by a classification scheme based on approximate momentum conservation in the center of the trap and near the Fermi energy. This is described in the following subsection.

\section{B. Classification of coupling coefficients}

We are interested in the modification of the zero temperature Fermi sea due to the interaction (11). This problem is different from that of scattering between excitations above the Fermi sea, which, in the present one-dimensional context, are probably not quasi particles. 
First of all, we must classify the coupling coefficients in Eq. (1). There are 24 coupling coefficients $V(m, p ; q, n)$ for any set of four distinct integers. Due to the symmetries $(m \leftrightarrow q),(n \leftrightarrow p)$, and $([m, q] \leftrightarrow[n, p])$ only three coupling coefficients remain. It is shown in [34] that among them, those with index combinations $n=m+p-q, n=p+q-m$, and $n=m+q-p$ dominate, provided the fermion number $N$ is large. It is here, where the fermionic nature of the quantum gas becomes relevant.

We can thus write

$$
V(m, p ; q, n) \rightarrow V_{a} \delta_{m-q, n-p}+V_{b} \delta_{q-m, n-p}+V_{c} \delta_{m+q, n+p} .
$$

Other weakly inhomogeneous trapping potentials also give dominant sets of $V_{a}, V_{b}$ and $V_{c}$ coupling constants but do not lead to a linear dispersion required for strict bosonization. Note that the coupling constants still depend on indices, e.g. $V_{c}=V(m, p ; q, n=m+q-p)$. In the present model, this is further simplified to $V_{c}=V_{c}(|p-q|)$.

Qualitatively, Eq. (6) can be understood as follows: The single particle states $\psi_{n}$ well inside the trap are superpositions of plane wave states $\exp \left(i k_{n} z\right)$ with $k_{n}= \pm \alpha \sqrt{2 n+1}$. For $N \gg 1$, the relevant states are near the Fermi energy and thus $\left|k_{n}\right| \approx k_{F}=\alpha \sqrt{2 N-1}$. Here, $k_{F}$ denotes the Fermi wave number.

According to Eq. (11), incoming states $\{n, q\}$ are transformed into $\{p, m\}$ in the collision process and the momenta of these states are (approximately, because of the weakly inhomogeneous trapping potential) conserved. Denoting a state with $k_{n} \approx-k_{F}$ by $(-n)$, three distinct collision processes can be discriminated:

$$
\{n, q\} \rightarrow\{p, m\}, \quad\{n,(-q)\} \rightarrow\{p,(-m)\}, \quad\{n,(-q)\} \rightarrow\{(-p), m\} .
$$

Processes with strict momentum conservation dominate: This explains the Kronecker symbols in the approximate relation (6). Momentum transfer in the first two cases is small, thus describing forward scattering. In the last case, the momentum transfer is about $2 k_{F}$, which corresponds to backward scattering. The first two cases were considered in [25, 26]. The last one requires an extension of the bosonization method, which is the aim of the present paper.

The couplings $V_{a}, V_{b}$, and $V_{c}$ are the analogues of the Luttinger model couplings $g_{4}, g_{2}$, and $g_{1}$, respectively 21, 23, 24]. In contrast to the Luttinger case, it was found in 34] that in a gas of identical fermions confined to the harmonic trap, forward scattering is almost completely suppressed so that backscattering is the dominant interaction process, unless the pair interaction potential is of long range. This is essentially a consequence of the Fermi algebra. In the following, we will ignore $V_{a}$ and $V_{b}$ completely though a more general treatment is possible.

In restricting the full set of interacting matrix elements to a set of solvable interactions we define a simplified model which cannot fully represent the initial problem.

For a number of properties such as anomalous dimensions, we can, however, expect universality in the sense of the Luttinger liquid phenomenology [21,23]. This is confirmed by our result for the one-particle correlation function (Sec. V B) which shows Luttinger liquid behavior in the center of the trap.

\section{BACKSCATTERING AND BOSONIZATION}

The treatment of forward scattering is described in [25, 26]. We give here only the extensions necessary for the inclusion of the backscattering interaction coefficients

$$
V(m, p ; q, n)=V_{c}(|q-p|) \delta_{m+q, n+p} .
$$

Substituting these interaction coefficients into Eq. (1) and reordering operators gives

$$
\hat{V}_{c}=-\frac{1}{2} \sum_{m p, v \neq 0} V_{c}(|v|)\left(\hat{c}_{m}^{\dagger} \hat{c}_{m+v}\right)\left(\hat{c}_{p}^{\dagger} \hat{c}_{p+v}\right)+\frac{1}{2} \sum_{m, v \neq 0} V_{c}(|v|)\left(\hat{c}_{m+v}^{\dagger} \hat{c}_{m-v}\right) .
$$

We omitted terms which are proportional to the fermion number operator by setting $V_{c}(0)=0$. The second term is a one-particle operator, which appears due to the backscattering. The perturbation Eq. (9) is exactly solvable. Thus there is no renormalization group flow of the coupling $V_{c}$.

The essential requirement of the bosonization method is the possibility to express all operators entirely in terms of density fluctuation operators. It is still met in the present case: Introducing the density fluctuation operators 


$$
\hat{\rho}(p) \equiv \sum_{q} \hat{c}_{q+p}^{\dagger} \hat{c}_{q}
$$

or, more conveniently, canonical boson operators related to them by:

$$
\hat{\rho}(p)=\left\{\begin{array}{lll}
\sqrt{|p|} & \hat{d}_{|p|}, & p<0 \\
\sqrt{p} & \hat{d}_{p}^{\dagger}, & p>0
\end{array}\right.
$$

it is found that bosonic commutation relations

$$
\left[\hat{d}_{m}, \hat{d}_{n}^{\dagger}\right]=\delta_{m, n}
$$

are satisfied after introducing the anomalous vacuum (cf. e.g., 24, 35]).

The bosonized form of the backscattering operator is

$$
\hat{V}_{c}=-\frac{1}{2} \sum_{m>0} m V_{c}(m)\left\{\hat{d}_{m}^{2}+\hat{d}_{m}^{\dagger 2}\right\}+\frac{1}{2} \sum_{m>0} \sqrt{2 m} V_{c}(m)\left[\hat{d}_{2 m}+\hat{d}_{2 m}^{\dagger}\right] .
$$

It is seen that the two-particle interaction due to backward scattering is of the same form as the forward scattering operator $\hat{V}_{b}$ studied in 25] except for a sign change, $V_{b} \rightarrow-V_{c}$. This is in complete analogy to the Luttinger case. However, the remaining one-particle operator produces non-trivial changes.

In order to diagonalize the total Hamiltonian

$$
\tilde{H}=\hbar \omega_{\ell} \sum_{m>0} m \hat{d}_{m}^{\dagger} \hat{d}_{m}+\hat{V}_{c}
$$

we perform two canonical transformations:

$$
\hat{d}_{m}=\hat{S}_{2}^{\dagger}\left\{\hat{S}_{1}^{\dagger} \hat{f}_{m} \hat{S}_{1}\right\} \hat{S}_{2}
$$

The first one

$$
\hat{S}_{1}=\exp \left[\frac{1}{2} \sum_{m>0} \zeta_{m}\left(\hat{f}_{m}^{2}-\hat{f}_{m}^{\dagger 2}\right)\right]
$$

is a kind of squeezing transformation and was used in 25] to diagonalize the two-particle interactions. It gives

$$
\left\{\hat{S}_{1}^{\dagger} \hat{f}_{m} \hat{S}_{1}\right\}=\hat{f}_{m} \cosh \zeta_{m}-\hat{f}_{m}^{\dagger} \sinh \zeta_{m}
$$

The second one

$$
\hat{S}_{2}=\exp \left[\sum_{m>0} \eta_{m}\left(\hat{f}_{m}^{\dagger}-\hat{f}_{m}\right)\right]
$$

is a displacement in order to get rid of the terms linear in the $\hat{d}$ and $\hat{d}^{\dagger}$ operators. The total result is

$$
\hat{d}_{m}=\hat{f}_{m} \cosh \zeta_{m}-\hat{f}_{m}^{\dagger} \sinh \zeta_{m}+\eta_{m} \exp \left(-\zeta_{m}\right)
$$

We find two diagonalization conditions: The standard one 


$$
\tanh 2 \zeta_{m}=-\frac{V_{c}(m)}{\hbar \omega_{\ell}}
$$

and another one due to backscattering

$$
\eta_{m}=\left\{\begin{array}{clrl}
-V_{c}(m / 2) \exp \left(-\zeta_{m}\right) /\left(2 \sqrt{m} \epsilon_{m}\right), & m=2 n, \\
0, & m=2 n-1 .
\end{array}\right.
$$

The final form of the Hamiltonian is

$$
\tilde{H}=\tilde{H}_{0}+\tilde{V}_{c}=\sum_{m>0} m \epsilon_{m} \hat{f}_{m}^{\dagger} \hat{f}_{m}+\text { const. . }
$$

The renormalized oscillator frequencies are

$$
\epsilon_{m} \equiv \sqrt{\left(\hbar \omega_{\ell}\right)^{2}-V_{c}^{2}(m)}
$$

and

$$
\exp \left(-\zeta_{m}\right) \equiv \sqrt{K_{m}}
$$

defines the dimensionless coupling constants

$$
K_{m} \equiv \sqrt{\frac{\hbar \omega_{\ell}+V_{c}(m)}{\hbar \omega_{\ell}-V_{c}(m)}} .
$$

\section{One-particle matrix elements}

We apply the theory to the evaluation of the one-particle matrix elements $\left\langle\hat{c}_{m}^{\dagger} \hat{c}_{n}\right\rangle$. To this order, we follow the steps in [25] and introduce the bosonic field

$$
\hat{\phi}^{\dagger}(v)=i \sum_{n=1}^{\infty} \frac{1}{\sqrt{n}} e^{-i n v} \hat{d}_{n}^{\dagger} \neq \hat{\phi}(v),
$$

which allows to express a particle number conserving bilinear product of auxiliary Fermi fields studied by Schönhammer and Meden 39]

$$
\hat{\psi}_{a}(v) \equiv \sum_{l=-\infty}^{\infty} e^{i l v} \hat{c}_{l}=\hat{\psi}_{a}(v+2 \pi)
$$

as

$$
\hat{\psi}_{a}^{\dagger}(u) \hat{\psi}_{a}(v)=G_{N}(u-v) \exp \left\{-i\left[\hat{\phi}^{\dagger}(u)-\hat{\phi}^{\dagger}(v)\right]\right\} \exp \{-i[\hat{\phi}(u)-\hat{\phi}(v)]\} .
$$

The quantity $G_{N}(u)$ in Eq. (28) is a distribution valued Fermi sum defined by

$$
G_{N}(u)=\sum_{l=-\infty}^{N-1} e^{-i l(u+i \epsilon)} .
$$

In order to evaluate expectation values of exponentials containing $\hat{\phi}$-operators, one must 
a) express the $\hat{d}$-operators in terms of the free $\hat{f}$ and $\hat{f}^{\dagger}$-operators,

b) apply the bosonic Wick theorem.

The Wick theorem refers to homogeneous linear combinations of $\hat{f}$ and $\hat{f}^{\dagger}$-operators. Due to backscattering, the operator $\hat{\phi}$ contains a c-number part $\phi_{c}$, which must be treated separately:

$$
\phi_{c}(u) \equiv-i C(-u)
$$

with

$$
C(u) \equiv \sum_{m=1}^{\infty} \xi_{2 m} \exp (-2 i m u)
$$

The quantities $\xi_{2 m}$ are given by

$$
\xi_{2 m} \equiv \eta_{2 m} \sqrt{\frac{K_{2 m}}{2 m}}=-V_{c}(m) \frac{K_{2 m}}{4 m \epsilon_{2 m}} .
$$

Following the steps in 25], the zero temperature expectation value of Eq. (28) becomes

$$
\left\langle\hat{\psi}_{a}^{\dagger}(u) \hat{\psi}_{a}(v)\right\rangle=G_{N}(u-v) \exp \left[-W_{1}(u, v)-W_{2}(u, v)\right]
$$

with $W_{1}$ given by

$$
W_{1}(u, v)=\sum_{m>0} \frac{2}{m}\left[\gamma_{m}-\alpha_{m} \cos m(u+v)\right]\{1-\cos m(u-v)\}
$$

The interaction parameters are

$$
\alpha_{m}=\frac{1}{2} \sinh 2 \zeta_{m}, \quad \gamma_{m}=\sinh ^{2} \zeta_{m}
$$

The contribution from the one-particle operator is:

$$
W_{2}(u, v)=C(-u)-C(u)+C(v)-C(-v)=4 i \sum_{m=1}^{\infty} \xi_{2 m} \sin m(u-v) \cos m(u+v)
$$

In order to obtain the matrix elements, a number of calculational steps are done, starting with the coordinate transformation $(u+v) / 2=t, u-v=s$. Using $W_{i}(u, v)=\tilde{W}_{i}(s, t)$ and $\tilde{W}_{i}(s \pm 2 \pi, t)=\tilde{W}_{i}(s, t \pm \pi)=\tilde{W}_{i}(s, t)$, $(i=1,2)$ and further symmetries, one obtains

$$
\left\langle\hat{c}_{n-p}^{\dagger} \hat{c}_{n+p}\right\rangle=\int_{-\pi}^{\pi} \frac{d t}{2 \pi} \cos p t \int_{-\pi}^{\pi} \frac{d s}{2 \pi} \exp \left[-\tilde{W}_{1}(s, t / 2)-\tilde{W}_{2}(s, t / 2)\right]\left\{\frac{\exp [i s(n-N+1)]}{1-\exp (i s-\epsilon)}\right\} .
$$

The distribution in curly brackets can be written as

$$
\left\{\frac{\exp [i s(n-N+1)]}{1-\exp (i s-\epsilon)}\right\}=-\left\{\frac{\sin \left(n-N+\frac{1}{2}\right) s}{2 \sin (s / 2)}\right\}+i\left[\frac{\cos \left(n-N+\frac{1}{2}\right) s}{2 \sin (s / 2)}\right]+\pi \delta_{2 \pi}(s)
$$

By defining

$$
\tilde{W}_{2}(s, t / 2) \equiv i f(s, t)
$$


the final result becomes

$$
\begin{aligned}
\left\langle\hat{c}_{n-p}^{\dagger} \hat{c}_{n+p}\right\rangle=\frac{1}{2} \delta_{p, 0}- & \int_{-\pi}^{\pi} \frac{d t}{2 \pi} \cos (p t) \\
& \times \int_{-\pi}^{\pi} \frac{d s}{2 \pi}\left\{\frac{\sin [(n-N+1 / 2) s-f(s, t)]}{2 \sin (s / 2)}\right\} \exp \left[-\tilde{W}_{1}(s, t / 2)\right] .
\end{aligned}
$$

Besides fixing the transformation parameters $\zeta_{m}$ in Eq. (20), the effect of backscattering appears in the argument of the sine via the function $f(s, t)$, which is explicitly given by

$$
f(s, t)=4 \sum_{m=1} \xi_{2 m} \sin (m s) \cos (m t) .
$$

Backscattering thus destroys the specific form of particle-hole symmetry found in [25] for forward scattering.

\section{VALIDITY OF THE THEORY}

We can state that our solution Eq. (40) is the exact result for $\mathrm{N}$ one-dimensional fermions confined to a harmonic trap, interacting via Eq. (9), and immersed in an anomalous vacuum of fermions filling all negative energy states. Thus we have to assess the role of the anomalous vacuum for the present finite size system. Intuitively, it is clear that its role decreases with increasing Fermi energy $\epsilon_{F} \propto N$, the energy region where interactions are most relevant.

We must, however, also consider the strength of the interaction. The dominant dimensionless coupling constant in Eq. (25) is $K_{1} \equiv K$, since $K_{m}$ decreases with increasing $m$. It is then seen that $K$ varies from zero to infinity when the physical coupling coefficient $V_{c}(1)$ varies from $-\hbar \omega_{\ell}$ to $\hbar \omega_{\ell}$. Values of $V_{c}(1)$ outside this range are physically inaccessible, as are coupling constants $g=g_{2}=g_{4}<-\pi \hbar v_{F}$ in the corresponding Luttinger model [21, 23, 24, 35]. Note that at the extreme values $V_{c}(1)= \pm \hbar \omega_{\ell}$ the renormalized excitation energy $\epsilon_{1}$ according Eq. (23) vanishes.

In the Luttinger model, $K \rightarrow \infty$ or $g \rightarrow-\pi \hbar v_{F}$ corresponds to the strongest physically allowed attraction. The compressibility vanishes and a phase separation occurs (cf. the discussion in [23]).

Our numerical results for the particle density in the present model show an increasing extension beyond the classical turning points, i.e., the density progressively leaks out of the trap for increasing $K \gg 1$. The interpretation of this effect is difficult due to the presence of the anomalous vacuum and the finiteness of $N$ : It is conceivable that an increasing interaction pulls more and more fermions out of the anomalous vacuum as indicated by studies of the fermion sum rule below.

Nevertheless, we can make the following statement about the validity of our bosonization scheme: For any fixed $\left|V_{c}\right| /\left(\hbar \omega_{\ell}\right)<1$, i.e., $0<K<\infty$, the error due to the anomalous vacuum can be made as small as wanted by increasing the physical particle number $N$. We call this asymptotically exact.

Though we do not have an analytic expression for the error at present, we know from our study of the fermion sum rule and the results presented below that the error decays fast with increasing $N$, probably in an exponential way.

The precise nature of the singular points $K=0$ and $K=\infty$ in the present finite system requires, however, further investigation. In assessing its relevance for interacting confined fermions, one must also consider the dependence of $V_{c}$ on the particle number. This in turn depends on the specific form of the interaction potential (cf. [34]). It thus seems that there is no simple and general limit $N \rightarrow \infty$ accompanied by a proper scaling of $\omega_{l}$ and the interaction parameters ("thermodynamic limit") for the interacting system.

We expect, however, that the region near the center of the trap $\left(|z| \ll L_{F} \propto \sqrt{N}\right)$ acquires properties of a homogeneous Luttinger liquid. This is demonstrated for the central pair correlation function studied in the following Section and for a reasonably large particle number $N=10^{3}$.

We also note that numerical investigation of the interaction coefficients in [34] show that increasing $N$ does not alter the dominance of $V_{c}$ over the forward scattering coefficients in a gas of identical fermions.

\section{A. Numerical Method}

For the purpose of the present investigation, it is sufficient to use a simplified model ("toy" model IM1 in [25]) by retaining only the terms with $v= \pm 1$ in Eq. (9). The relevant parameter then are: 


$$
\alpha_{1}=\frac{1-K^{2}}{4 K}, \quad \gamma_{1}=\frac{(1-K)^{2}}{4 K}
$$

The function $\tilde{W}_{1}(s, t / 2)$ in Eq. (40) becomes $2\left[\gamma_{1}-\alpha_{1} \cos (t)\right][1-\cos (s)]$ and the backscattering expression (41) simplifies to $f(s, t)=-V_{c}(1) \sin (s) \cos (t) / \hbar \omega_{\ell}$. The one-particle matrix elements at zero temperature are then given by

$$
\begin{aligned}
\left\langle\hat{c}_{M-p}^{\dagger} \hat{c}_{M+p}\right\rangle=\frac{1}{2} \delta_{p, 0}-\int_{\pi}^{\pi} \frac{d t}{2 \pi} \cos (p t) & \int_{-\pi}^{\pi} \frac{d s}{2 \pi}\left\{\frac{\sin [(M+1 / 2-N) s-f(s, t)]}{2 \sin (s / 2)}\right\} \\
& \times \exp \left\{-2\left[\gamma_{1}-\alpha_{1} \cos (t)\right][1-\cos (s)]\right\}
\end{aligned}
$$

This is the prediction of the bosonization method for identical fermions in the one-dimensional harmonic trap with dominant backscattering.

In [25], it was already pointed out that first order perturbation theory in fermionic Hilbert space reproduces the results using the equation corresponding to Eq. (43) for forward scattering $(f=0)$ when it is expanded to first order in $\alpha_{1}$ and $\beta_{1}$, and the same applies to the present case: Both approaches lead to the weak coupling result

$$
\left\langle\hat{c}_{M-p}^{\dagger} \hat{c}_{M+p}\right\rangle=\delta_{p, 0} \Theta(N-M-1 / 2)-\left(\frac{V_{c}(1)}{2 \hbar \omega_{\ell}}\right) \delta_{M, N-1}\left(\delta_{p, 1}+\delta_{p,-1}\right)+O\left(\frac{V_{c}(1)}{\hbar \omega_{\ell}}\right)^{2}
$$

Finally, the predictions of Eq. (43) will be checked against numerical results obtained by direct diagonalization in the fermionic $N$-particle Hilbert space and for strong coupling.

The fermionic $N$-particle Hilbert space is spanned by the unperturbed $N$-particle states

$$
|\{m\}\rangle^{(0)}=\hat{c}_{m_{1}}^{\dagger} \hat{c}_{m_{2}}^{\dagger} \cdots \hat{c}_{m_{N}}^{\dagger}|v a c\rangle
$$

Here, $\{m\}$ denotes a sequence of occupation numbers $m_{n}=0,1$ for the single particle states $\psi_{n}(z)$. For example, the three-particle state with energy $(11 / 2) \hbar \omega_{\ell}$ and excitation energy $\hbar \omega_{\ell}$ is

$$
|1,1,0,1,0,0, \ldots\rangle^{(0)}=\hat{c}_{0}^{\dagger} \hat{c}_{1}^{\dagger} \hat{c}_{3}^{\dagger}|v a c\rangle
$$

In order to simplify the notation for the unperturbed $N$-particle states, we classify them according to their excitation energies $\Delta E(n)=n \hbar \omega_{\ell}$. Degeneracy is taken care of by ordering the states according to the lowest unoccupied single particle state occurring in them. They are then numbered consecutively $|m\rangle^{(0)}, m=0,1,2, \ldots$ The unperturbed ground state is thus

$$
|0\rangle^{(0)}=|1,1, \ldots, 1,1,0,0, \ldots\rangle
$$

and subsequent excited states are

$$
\begin{aligned}
|1\rangle^{(0)} & =|1,1, \ldots, 1,0,1,0,0, \ldots\rangle, \\
|2\rangle^{(0)} & =|1,1, \ldots, 0,1,1,0,0, \ldots\rangle, \\
|3\rangle^{(0)} & =|1,1, \ldots, 1,0,0,1,0,0, \ldots\rangle, \quad \text { etc.. }
\end{aligned}
$$

The perturbed ground state to first order used to obtain Eq. (44) is

$$
|0\rangle^{(1)}=|0\rangle^{(0)}+\left(\frac{V_{c}(1)}{2 \hbar \omega_{\ell}}\right)|2\rangle^{(0)} .
$$


This result was also used to check the numerical procedure.

The actual eigenstates of the interacting Hamiltonian are denoted by $|s\rangle$. They are expanded according to

$$
|s\rangle=\sum_{m} c_{s m}|m\rangle^{(0)}
$$

These expansion coefficients are the central quantities in the numerical procedure: In terms of the expansion coefficients, the occupation probabilities for oscillator states $\psi_{M}$ with respect to the ground state $|0\rangle$ are

$$
P(M)=\sum_{m n} c_{0 m} c_{0 n}^{*}{ }^{(0)}\left\langle n\left|\hat{c}_{M}^{\dagger} \hat{c}_{M}\right| m\right\rangle^{(0)}
$$

Similarly, the particle density, which is the expectation value of the density operator $\hat{\psi}^{\dagger}(z) \hat{\psi}(z)$ with $\hat{\psi}(z) \equiv$ $\sum_{n} \psi_{n}(z) \hat{c}_{n}$, becomes

$$
n(z)=\left\langle 0\left|\hat{\psi}^{\dagger}(z) \hat{\psi}(z)\right| 0\right\rangle=\sum_{p=0, q=0}^{\infty} \psi_{p}(z) \psi_{q}(z) \sum_{m n} c_{0 m} c_{0 n}^{*}{ }^{(0)}\left\langle n\left|\hat{c}_{p}^{\dagger} \hat{c}_{q}\right| m\right\rangle^{(0)} .
$$

The first computational step is the evaluation of the interaction matrix elements

$$
V_{i j}={ }^{(0)}\left\langle i\left|\hat{V}_{c}\right| j\right\rangle^{(0)} .
$$

In detail, the free $N$-particle eigenstates Eq. (45) and the expectation values in Eqs.(51), (152), and (53) with respect to these states are calculated via an algorithm which implements the fermion-algebra. The number of eigenstates which are taken into account grows strongly with the maximal excitation energy $\Delta E\left(n_{\max }\right)$ due to increasing degeneracy.

In subsequent steps, the diagonalization of the matrix

$$
H_{i j}=H_{0 i j}+V_{i j},
$$

and the computation of the expansion coefficients $c_{s m}$ follows [36, 37]. Eigenvalues and eigenvectors of the matrix (54) are computed using a QL algorithm. The normalized eigenvector of the ground state determines the required expansion coefficients $c_{0 m}$ according to ${ }^{(0)}\langle m \mid 0\rangle$.

\section{B. Occupation Probabilities}

The occupation probabilities of oscillator states $\psi_{M}$ in the interacting ground state are

$$
0 \leq P(M)=\left\langle 0\left|\hat{c}_{M}^{\dagger} \hat{c}_{M}\right| 0\right\rangle \leq 1 .
$$

Due to the backward scattering, they do not show the symmetry found in [25, 26] for forward scattering.

We will also discuss the sum rule

$$
S\left(N, \alpha_{1}\right) \equiv \sum_{m=0}^{\infty}\left\langle\hat{c}_{m}^{\dagger} \hat{c}_{m}\right\rangle \stackrel{?}{=} N
$$

for the fermion number. This sum rule is only asymptotically fulfilled in the bosonization method: The particles in the anomalous vacuum couple to the physical particles, giving an effective particle number $S\left(N, \alpha_{1}\right)>N[\underline{38}$. The effect is most pronounced when the particle number $N$ is smaller than the coupling strength $\left|\alpha_{1}\right|$. The coupling to the anomalous vacuum also leads to the surprising feature that interaction effects occur for just one physical particle $(N=1)$. 


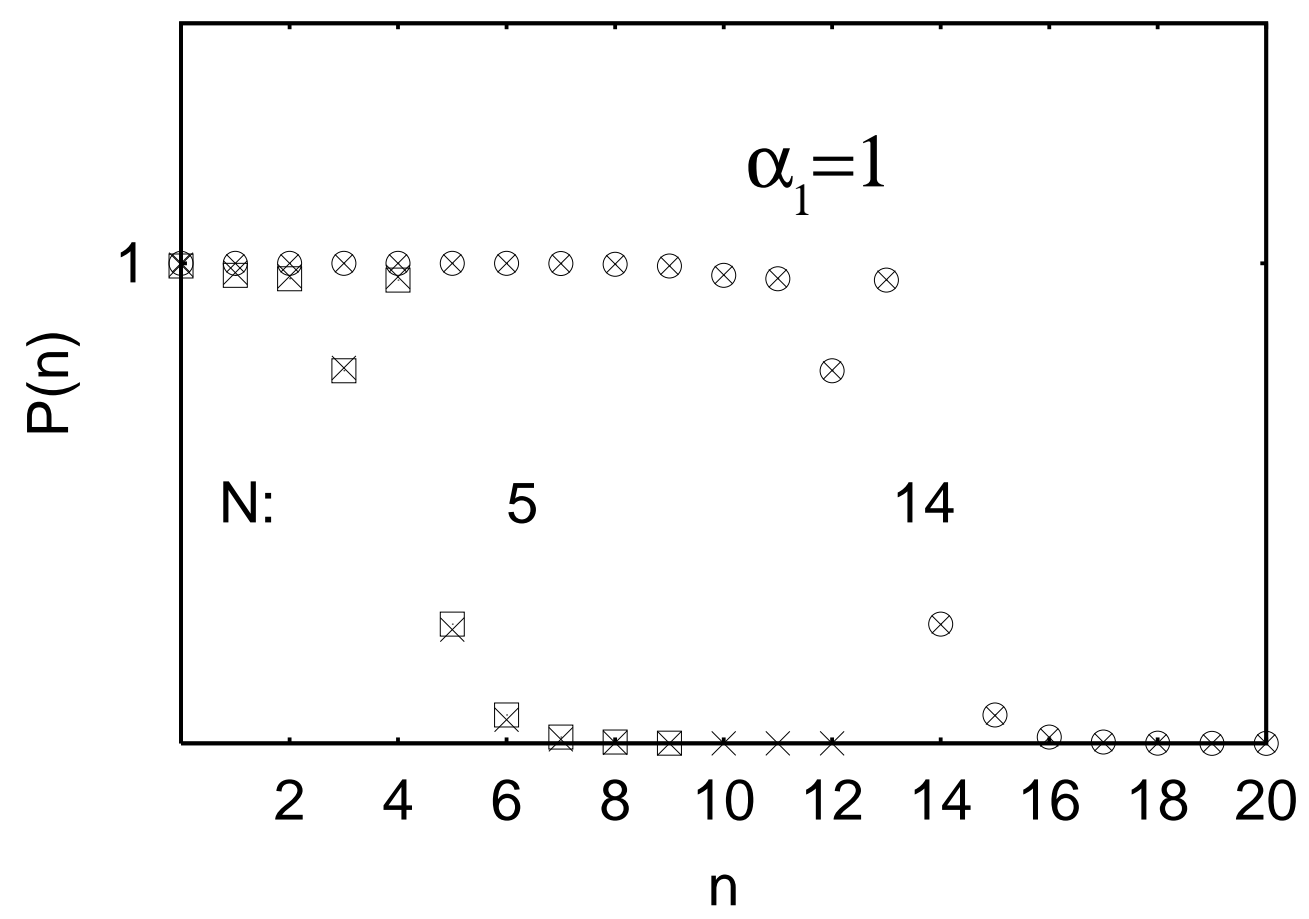

Fig. 1: Occupation probabilities $P(n)$ of oscillator states $\psi_{n}$ in the interacting system for repulsive backscattering strength $\alpha_{1}=1$ at zero temperature and particle numbers $N=5$ and $N=14$. Crosses are results of numerical diagonalization, predictions of the bosonization method are given as squares $(N=5)$ and circles $(N=14)$, respectively. No significant deviations between the two approaches are seen for particle number $N=14$. For $N=5$, the presence of the anomalous vacuum in the bosonization method causes visible deviations.

Figure 1 shows a comparison of occupation probabilities calculated using the bosonization method and numerical diagonalization. For a coupling constant $\alpha_{1}=1$, which corresponds to $V_{c}(1)=-0.894 \hbar \omega_{\ell}$, no significant deviations are found for particle numbers $N=14$ and larger. However, for $N=5$, the bosonization method is less accurate because the anomalous vacuum is present. Though the excess $\Delta N(5,1) \equiv S(5,1)-N$ amounts to only $5.4 \cdot 10^{-4}$, individual occupation probabilities are less accurate.

\section{Particle density}

The density of the trapped particles can be written as

$$
n(z)=\sum_{M=0}^{\infty} \psi_{M}(z)^{2}\left\langle\hat{c}_{M}^{\dagger} \hat{c}_{M}\right\rangle+2 \sum_{M=1}^{\infty} \sum_{p=1}^{M} \psi_{M-p}(z) \psi_{M+p}(z)\left\langle\hat{c}_{M-p}^{\dagger} \hat{c}_{M+p}\right\rangle .
$$

The particle density shows Friedel oscillations [40, 41]. The amplitudes of the Friedel oscillations are modified by the interaction as described in [25, 26, 43].

Figure 2 displays particle densities for the coupling value $\alpha_{1}=10$, corresponding to $V_{c}(1)=-0.998 \hbar \omega_{\ell}$, which is a rather large repulsive interaction. The results of numerical diagonalization and of the bosonization method are compared for various particle numbers $N$. The deviations found are due to the presence of the anomalous vacuum in the bosonization method which interacts with the physical particles. The differences are more pronounced for larger coupling strength or smaller particle number $N$ : Larger particle numbers lead to larger Fermi energies $\epsilon_{F}$. This decouples the physically relevant energy region $\epsilon \approx \epsilon_{F}$ from the anomalous vacuum. Larger coupling strengths counteract the decoupling. 


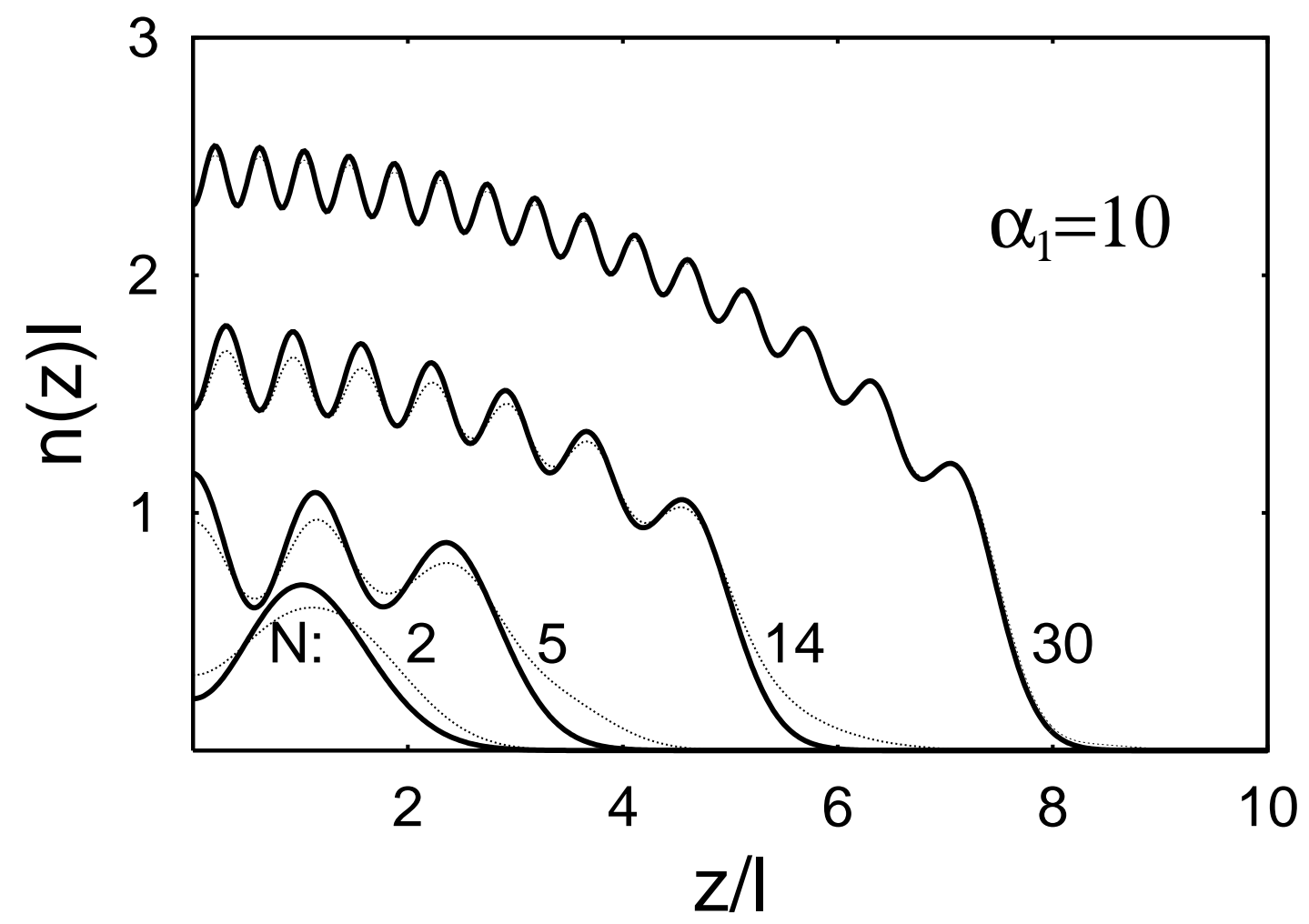

Fig. 2: Dimensionless particle density $n(z) l$ in units of the inverse of the oscillator length $l$ versus dimensionless distance $z / l$ from the center of the one-dimensional harmonic trap for several particle numbers $N$ of a Fermi gas with dominant backward scattering at zero temperature. Thick curves are from numerical diagonalization and dotted curves show the Friedel oscillations calculated by the bosonization method. Interaction strength is $\alpha_{1}=10$. Deviations for particle numbers less than $N=30$ are due to the anomalous vacuum, which couples strongly to the real particles.

We can summarize: The results of the bosonization method for the one-particle matrix elements of a harmonically trapped one-dimensional Fermi gas with backscattering when compared with the exact numerical diagonalization show noticeable deviations from the exact results when the physical number $N$ of fermions is small or the interaction strength is large. This effect is not restricted to backscattering. Forward scattering would produce similar results. For sufficiently large $N$, the bosonization method produces, however, fully acceptable results. It is then far more effective than any numerical approach.

\section{RESULTS FOR LARGE N}

The functions $\tilde{W}_{1}(s, t)$ and $f(s, t)$ in the exact expression (40) for the matrix elements contain a bewildering number of interaction coefficients, which all depend on the index $m$. In order to proceed with the evaluation, we describe in the Appendix a model for the interaction coefficients, which allows to do all summations and express them by just two parameters: The main coupling constant $K$ and a small number $r \ll 1$ specifying the exponential decay of all interaction coefficients. Following [26], we adopt $r=1 / \sqrt{N}$ so that only the coupling constant $K>0$ remains. $K>1$ corresponds to attractive interactions and $0<K<1$ to repulsion between the fermions. An analogous procedure is used in the theory of the Luttinger model [21, 22, 23, 24].

The result for the one-particle matrix elements becomes

$$
\begin{aligned}
\left\langle\hat{c}_{n-p}^{\dagger} \hat{c}_{n+p}\right\rangle=\frac{\delta_{p, 0}}{2}- & \int_{-\pi}^{\pi} \frac{d t}{2 \pi} \frac{\cos (p t)}{\left[1+Z_{\alpha}-\cos (t)\right]^{\alpha_{0}}} \int_{-\pi}^{\pi} \frac{d s}{2 \pi}\left\{\frac{\sin [(n-N+1 / 2) s-f(s, t)]}{2 \sin (s / 2)}\right\} \\
& \times\left\{\frac{Z_{\gamma}}{\left[1+Z_{\gamma}-\cos (s)\right]}\right\}^{\gamma_{0}}\left\{\left[1+Z_{\alpha}-\cos (t-s)\right]\left[1+Z_{\alpha}-\cos (t+s)\right]\right\}^{\alpha_{0} / 2},
\end{aligned}
$$


with constants $Z_{\gamma}$ and $Z_{\alpha}$ according to

$$
Z_{\gamma}=\cosh \left(r_{\gamma}\right)-1 \rightarrow r^{2} / 2, \quad Z_{\alpha}=\cosh \left(r_{\alpha} / 2\right)-1 \rightarrow r^{2} / 8
$$

The coupling dependent exponents in Eq. (58) are

$$
\alpha_{0}=\frac{1}{4 K}\left(1-K^{2}\right), \quad \gamma_{0}=\frac{1}{4 K}(1-K)^{2} \geq 0 .
$$

The sign of $\alpha_{0}$ is negative when the backscattering is attractive.

In addition, we take advantage of the fact that backscattering is dominant in a harmonically confined gas of identical fermions: The function $f$ is then determined by the backscattering contribution $C(u)$ in Eq. A13):

$$
f(s, t)=2 \Im[C(t / 2-s / 2)-C(t / 2+s / 2)]=\frac{1}{4}\left(1-K^{2}\right) \arctan \left[\frac{2 q(\cos t-q \cos s) \sin s}{1-q^{2}-2 q(\cos t-q \cos s) \cos s}\right]
$$

with

$$
q=\exp (-r / 2) \rightarrow 1-r / 2+r^{2} / 8
$$

\section{A. Particle and momentum densities}

The particle density $n(z)$ is evaluated using Eq. (57) and the matrix elements Eq. (58). For large $N$ and moderate coupling ( $K$ is of order unity), the particle density well inside the trap is dominated by the large number of nearly filled states inside the Fermi sea. However, the states above the Fermi level $N_{F}=N-1$ make significant contributions to $n(z)$ by modifying the Friedel oscillations [40]: This is demonstrated in Fig. 3 using the full expression (58).

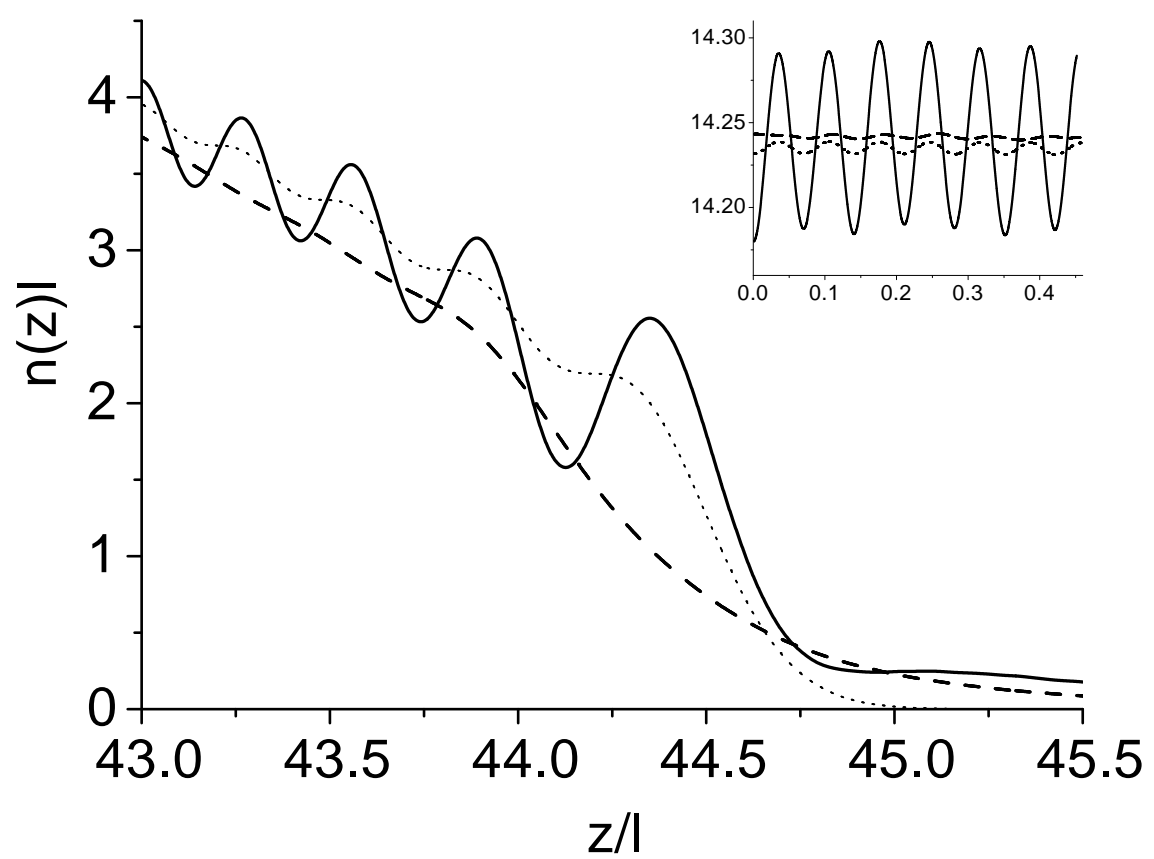

Fig. 3: Dimensionless particle density $n(z) l$ in units of the inverse of the oscillator length $l$ versus dimensionless distance $z / l$ near the classical boundary at $L_{F}=l \sqrt{2 N-1}$ of the one-dimensional harmonic trap for $N=1000$ interacting spinless fermions at zero temperature. Dotted curve shows unperturbed Friedel oscillations. Strongly 
oscillating curve refers to a repulsive interaction with $K=1 / 3$ while the dashed curve is for an attractive interaction with $K=3$. The inset displays the density near the center of the trap, where Friedel oscillation have the usual period $\pi / k_{F}=\pi l / \sqrt{2 N-1}$.

It is seen that Friedel oscillations are enhanced in the repulsive case while an attractive interaction suppresses them. Near the classical boundary $|z| \approx L_{F}=l \sqrt{2 N-1}$, the oscillation period is larger than the standard value $\pi / k_{F}$ well inside the trap and obeys the relations found in [41] for the non-interacting case. Furthermore, it is seen that the perturbed density extends considerably into the classically forbidden region. The effect becomes stronger with increasing coupling strength $(K \gg 1$ or $\ll 1)$.

The momentum density distribution is given by

$$
p(k)=l^{2} \sum_{n=0}^{\infty} \sum_{p=-n}^{n}(-1)^{p} \psi_{n-p}\left(k l^{2}\right) \psi_{n+p}\left(k l^{2}\right)\left\langle\hat{c}_{n-p}^{\dagger} \hat{c}_{n+p}\right\rangle
$$

It is seen that $p(k)$ is identical in shape to the density distribution $n(z)$ provided the diagonal approximation for the one-particle matrix elements is reasonable. This is the case for moderate attractive coupling.

We know [25] that the Friedel oscillations in $n(z)$ and $p(k)$ behave oppositely: Attractive interactions increase them in the momentum density and decrease them in the particle density and vice versa for repulsion. A corresponding effect is seen in the Wigner function discussed below.

\section{B. Wigner function}

In terms of the local creation and annihilation operators $\hat{\psi}^{\dagger}(z)$ and $\hat{\psi}(z)$, the static Wigner function of the manyfermion system is given (cf. [42]) by

$$
W(z, k)=\int_{-\infty}^{\infty} d \zeta e^{-i k \zeta}\left\langle\hat{\psi}^{\dagger}(z-\zeta / 2) \hat{\psi}(z+\zeta / 2)\right\rangle
$$

The Wigner function of the one-dimensional Fermi gas with two components and forward scattering between the two components was studied in [43].

Transforming to the oscillator representation gives

$$
W(z, k)=\sum_{m, n=0}^{\infty}\left\langle\hat{c}_{m}^{\dagger} \hat{c}_{n}\right\rangle f_{m n}(z, k),
$$

with expansion coefficients $f_{m n}$ according to

$$
f_{m n}(z, k)=\int_{-\infty}^{\infty} d \zeta e^{-i k \zeta} \psi_{m}(z-\zeta / 2) \psi_{n}(z+\zeta / 2)=f_{n m}(z,-k)
$$

These are explicitly given in terms of generalized Laguerre polynomials ( $n \geq m$, cf. e.g., [44]) by

$$
f_{m n}(z, k)=2(-1)^{m}\left(2^{n-m} m ! / n !\right)^{1 / 2}(z / l-i k l)^{n-m} \exp \left(-z^{2} / l^{2}-k^{2} l^{2}\right) \mathrm{L}_{m}^{(n-m)}\left(2 z^{2} / l^{2}+2 k^{2} l^{2}\right) .
$$

It is noted that the one-particle matrix elements can be completely reconstructed from the Wigner function:

$$
\left\langle\hat{c}_{m}^{\dagger} \hat{c}_{n}\right\rangle=\frac{1}{2 \pi} \int_{-\infty}^{\infty} d z d k f_{n m}(z, k) W(z, k)
$$

The Wigner function is thus equivalent to the full set of one-particle matrix elements.

We show an example of the Wigner function in Figs. 4(a) and 4(b) for a repulsive interaction. 

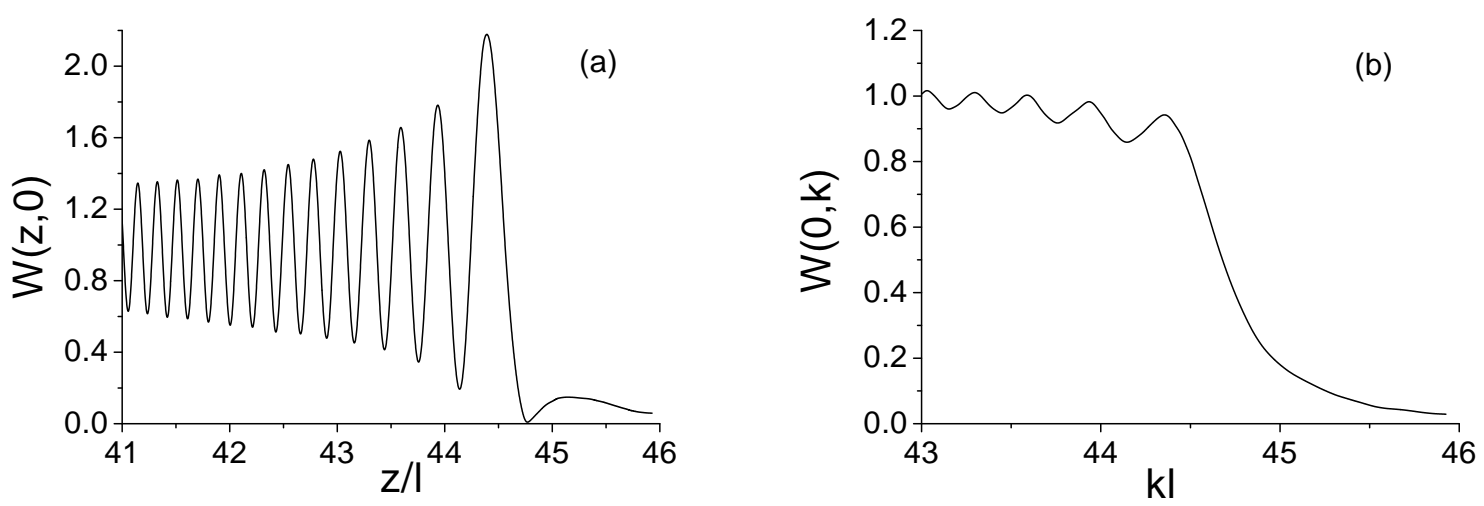

Fig. 4: Friedel oscillations in phase space: Wigner function for $N=1000$ interacting spinless fermions at zero temperature in the one-dimensional harmonic trap. In (a), the cross section $W(z, k=0)$ is plotted versus dimensionless distance $z / l$ displaying the region around the classical turning point $L_{F}=l \sqrt{2 N-1}$. 1 is the oscillator length. In (b), the corresponding function $W(z=0, k)$ is shown versus dimensionless momentum $k l$. Note the increase of the oscillation amplitude near the classical boundary. The plot refers to a repulsive interaction with $K=1 / 3$. Repulsive interactions enhance Friedel oscillations in the spatial direction and suppress them in the momentum direction.

The amplitudes of the Friedel oscillations increase near the classical turning point. This was discussed for the non-interacting case [41].

Neglecting non-diagonal matrix elements in Eq. 655) would give $W(z, k=0)=W\left(z=0, k \rightarrow z / l^{2}\right)$. The significant differences between Fig. 4(a) and Fig. 4(b) thus stem from the non-diagonal matrix elements, which are particular relevant for repulsive interactions.

The static pair correlation function with respect to the center of the trap

$$
C\left(z, z^{\prime}=0\right) \equiv\left\langle\hat{\psi}^{\dagger}(z) \hat{\psi}\left(z^{\prime}=0\right)\right\rangle=\frac{1}{2 \pi} \int_{-\infty}^{\infty} d k e^{-i k z} W(z / 2, k),
$$

as well as any other one-particle property of the many-particle system are also contained in the Wigner function.

The central pair correlation function (69) is displayed in Fig. 5. It is noted that the wave length of the intrinsic periodicity in the center of the trap is twice that of the Friedel oscillations, i.e., $\lambda=2 \pi / k_{F}$. In fact, the central pair correlation function for non-interacting fermions is given by

$$
C_{0}\left(z, z^{\prime}=0\right)=\frac{1}{l \sqrt{\pi}} e^{-z^{2} /\left(2 l^{2}\right)}\left[\sum_{n=0}^{M}(-1)^{n} \frac{H_{2 n}(z / l)}{4^{n} n !}\right] \rightarrow \frac{\sin \left(k_{F} z\right)}{\pi z},
$$

provided $N=2 M$ is large and $z$ is restricted to the center of the trap $\left(|z| \ll L_{F}\right)$. We expect that interactions modify Eq. (70) according to

$$
C\left(l \ll z \ll L_{F}, z^{\prime}=0\right) \propto \frac{\sin \left(k_{F} z\right)}{l(z / l)^{\alpha_{C}}},
$$

i.e., the anomalous dimension of the correlation function is $\alpha_{C}=1+2 \gamma_{0}=(K+1 / K) / 2$, as in a Luttinger liquid. This is confirmed numerically by displaying the corresponding envelope in Fig. 5. 


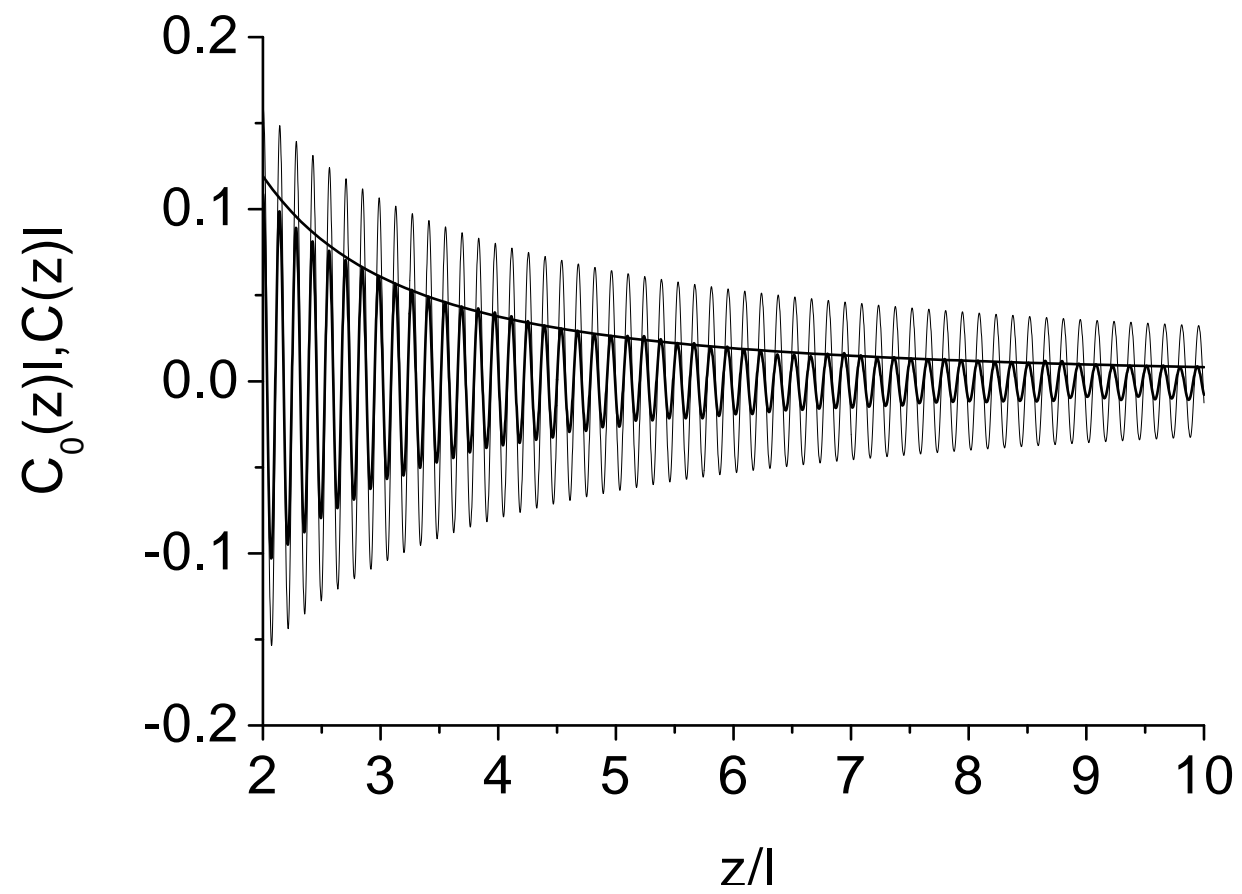

Fig. 5: Dimensionless correlation functions $C_{0}\left(z, z^{\prime}=0\right) l$ and $C\left(z, z^{\prime}=0\right) l$ in units of the inverse of the oscillator length $l$ versus dimensionless distance $z / l$ from the center of the trap for $N=1000$ spinless fermions at zero temperature. Thin curve shows non-interacting function $C_{0}$ while the thick curve gives $C$ for an attractive interaction with $K=3$. Oscillations have twice the period of Friedel oscillations. Envelope to the full curve displays power law decay with anomalous dimension $(K+1 / K) / 2$.

The correlation function $C$ of the interacting system thus decays faster than $C_{0}$ and this effect is invariant under $K \rightarrow 1 / K$.

\section{SUMMARY}

The fact that backscattering dominates the interaction between identical one-dimensional fermions confined to a harmonic trap makes it necessary to include backscattering into the bosonization method. This can be done exactly by supplementing the squeezing transformation (16) with the displacement transformation (18). This changes (in fact complicates) the result (40) for the one-particle matrix elements by altering the argument in the sine-function. This modification destroys the symmetry

$$
\left\langle\hat{c}_{2 N-1-n-p}^{\dagger} \hat{c}_{2 N-1-n+p}\right\rangle=\delta_{p, 0}-\left\langle\hat{c}_{n-p}^{\dagger} \hat{c}_{n+p}\right\rangle .
$$

In order to evaluate the new result, we introduce in analogy to the Luttinger model a simplified form for the interaction coefficients which uses only one coupling constant $K . K=1$ corresponds to the non-interacting case studied in 41, 45, 46, 47], $K>1$ corresponds to attraction. For values of $K$ near unity, the Fermi edge at zero temperature is already significantly smoothed out by the interactions, an effect which becomes more pronounced for stronger coupling.

We then study particle and momentum densities for various coupling strengths. Friedel oscillations in phase space are characteristic for these quantities, which extend progressively into the classically forbidden region when the coupling strength is increased. The results confirm an earlier finding that attractive interactions decrease the amplitude of the Friedel oscillations in real space while repulsion enhances it. The Friedel oscillations in momentum space behave oppositely.

Finally, the central pair correlation function is calculated. The basic period of the pair correlation function in the center of the trap is $2 \pi / k_{F}$ in contrast to $\pi / k_{F}$ of the Friedel oscillations. Interactions do not affect these basic periodicities: Even the increase of the Friedel period in the particle density near the classical boundaries is correctly 
given by the non-interacting theory. Interactions, however, modify the power law decay of the correlation function inside the trap. Our numerical results are consistent with the value

$$
\alpha_{C}=\frac{K+1 / K}{2}
$$

for the anomalous dimension $\alpha_{C}$ of the correlation well inside the trap, in accordance with the predictions for a Luttinger liquid.

We also assessed the validity of the bosonization method, comparing its result with those of direct numerical diagonalization in fermionic Hilbert space.

\section{Acknowledgments}

We gratefully acknowledge helpful discussions with G. Alber, T. Pfau, and W. P. Schleich and financial help by Deutsche Forschungsgemeinschaft.

\section{APPENDIX}

\section{A model for the interaction coefficients}

The result (40) for the one-particle matrix elements contains three sets of interactions coefficients: $\left\{\alpha_{m}\right\},\left\{\gamma_{m}\right\}$, and $\left\{V_{c}(m)\right\}$. The first two sets depend on the basic interaction coefficients $V_{c}(m)$ via the parameter $\zeta_{m}$ according to Eq. (20). The interaction coefficients $V_{c}(m)$ appear also directly in $W_{2}$. In order to get explicit results for the matrix elements, one must be able to perform the summations in $\tilde{W}_{1}$ and $\tilde{W}_{2}$. This requires models for the $m$-dependence of the above interaction coefficients.

Defining $\tilde{V}_{c} \equiv V_{c} / \hbar \omega_{\ell}$, an explicit form for $\alpha_{m}$ is

$$
\alpha_{m}=-\frac{\tilde{V}_{c}(m)}{2 \sqrt{\left[1-\tilde{V}_{c}(m)\right]^{2}}} \equiv \frac{1-K_{m}^{2}}{4 K_{m}} .
$$

Similarly, the central coupling constants $K_{m}$ determine $\gamma_{m}$ and the renormalized oscillator energies $\epsilon_{m}$ according to

$$
\gamma_{m}=\frac{\left(1-K_{m}\right)^{2}}{4 K_{m}}, \quad \epsilon_{m}=\hbar \omega_{\ell} \frac{2 K_{m}}{K_{m}^{2}+1} .
$$

Following the procedure in the Luttinger model, we adopt exponential decays, thus we make the ansatz:

$$
\alpha_{m}=\alpha_{0} \exp \left(-r_{\alpha} m / 2\right)
$$

and

$$
\gamma_{m}=\gamma_{0} \exp \left(-r_{\gamma} m\right)
$$

Since the signs of $\tilde{V}_{c}(m)$ do not depend on $m, \alpha_{m}$ is related to $\gamma_{m}$ by

$$
\alpha_{m}=-\operatorname{sgn}\left(\tilde{V}_{c}\right) \sqrt{\gamma_{m}\left(1+\gamma_{m}\right)}
$$

and one finds

$$
\alpha_{m}=-\operatorname{sgn}\left(\tilde{V}_{c}\right) \exp \left(-r_{\gamma} m / 2\right) \sqrt{\gamma_{0}\left[1+\gamma_{0} \exp \left(-r_{\gamma} m\right)\right]} .
$$


Assuming that $r_{\gamma}$ is very small, $0<r_{\gamma} \ll 1$, and that the relevant values of $m$ obey $m<1 / r_{\gamma}$, Eq. (A66) leads to

$$
\alpha_{m} \approx \exp \left(-r_{\gamma} m / 2\right) \alpha_{0}
$$

with

$$
\alpha_{0}=-\operatorname{sgn}\left(\tilde{V}_{c}\right) \sqrt{\gamma_{0}\left(1+\gamma_{0}\right)}
$$

Thus, we can set

$$
r_{\alpha}=r_{\gamma} \equiv r
$$

Another set of coupling parameters involves directly the backscattering coefficients $\tilde{V}_{c}(m)$ via $\xi_{2 m}$ in $\tilde{W}_{2}$. Using Eqs. (23) and (25), Eq. (32) becomes

$$
\xi_{2 m}=-\frac{\tilde{V}_{c}(m)}{4 m\left[1-\tilde{V}_{c}(2 m)\right]} .
$$

We take advantage of the slow decay of interaction coefficients with $m$ and write

$$
\xi_{2 m}=-\frac{1}{4 m} \tilde{V}_{c e f f} \exp \left(-r_{c} m\right)
$$

with another decay constant $r_{c} \ll 1$ and with

$$
\tilde{V}_{c e f f} \approx \frac{\tilde{V}_{c}(1)}{1-\tilde{V}_{c}(1)} \equiv \frac{1}{2}\left(K^{2}-1\right) .
$$

This gives a useful result for the backscattering function (31)

$$
C(u)=-\frac{\tilde{V}_{c e f f}}{4} \sum_{m=1}^{\infty} \frac{1}{m} \exp \left[\left(-m\left(2 i u+r_{c}\right)\right]=\frac{\tilde{V}_{c e f f}}{4} \ln \left[1-\exp \left(-r_{c}-2 i u\right)\right]\right.
$$

It is noted that consistency requires $\left|\tilde{V}_{c}\right| \leq 1$ and $K \geq 0$.

Comparing $\alpha(m)$ in Eq. (A1) with Eqs. (A10) and (A11), leads to

$$
\alpha_{m}=-\tilde{V}_{c e f f} \exp \left(-r_{c} m\right)\left\{\frac{1-\tilde{V}_{c}(2 m)}{2 \sqrt{1-\tilde{V}_{c}^{2}(m)}}\right\} .
$$

Suppressing the weak $m$-dependence in the curly brackets, i.e.,

$$
\tilde{V}_{c}(m) \rightarrow \tilde{V}_{c}
$$

allows the identifications

$$
r_{c}=\frac{r_{\alpha}}{2}=\frac{r}{2}
$$

Furthermore, using 


$$
\alpha_{0} \equiv-\frac{\tilde{V}_{c}}{2 \sqrt{1-\tilde{V}_{c}^{2}}}
$$

gives

$$
\tilde{V}_{c e f f}=-2 \sqrt{\frac{1+\tilde{V}_{c}}{1-\tilde{V}_{c}}} \alpha_{0}
$$

The main coupling constant

$$
K=\sqrt{\frac{1+\tilde{V}_{c}}{1-\tilde{V}_{c}}}
$$

determines all relevant couplings and the renormalized energy according to

$$
\tilde{V}_{c}=\frac{K^{2}-1}{K^{2}+1}, \quad \alpha_{0}=-\frac{K^{2}-1}{4 K}, \quad \gamma_{0}=\frac{(K-1)^{2}}{4 K}, \quad \epsilon=\hbar \omega_{\ell} \frac{2 K}{K^{2}+1} .
$$

Only the two parameters $K$ and $r=r_{\alpha}=r_{\gamma}=2 r_{c}$ remain. $K$ is determined by the physical backscattering strength $\tilde{V}_{c}(1) \equiv \tilde{V}_{c}$ according to Eq. A19. In 26], it was argued that $r=1 / \sqrt{N}$ is a reasonable choice for the decay constant. Finally, it is noted that interactions always decrease the renormalized excitation energy $\epsilon$ below the non-interacting value $\hbar \omega_{\ell}$.

[1] M. H. Anderson et al., Science 269, 198 (1995); K. B. Davis et al., Phys. Rev. Lett. 75, 3969 (1995); C. C. Bradley et al., ibid. 75, 1687 (1995).

[2] F. Brosens, J. T. Devreese, and L. F. Lemmens, Phys. Rev. E 57, 3871 (1998).

[3] G. M. Bruun and K. Burnett, Phys. Rev. A 58, 2427 (1998).

[4] M. A. Zaluska-Kotur, M. Gajda, A. Orlowski, and J. Mostowski, Phys. Rev. A 61, 033613 (2000).

[5] M. Houbiers et al., Phys. Rev. A 56, 4864 (1997).

[6] M. A. Baranov and D. S. Petrov, Phys. Rev. A 58, R801 (1998).

[7] M. Houbiers and H. T. C. Stoof, Phys. Rev. A 59, 1556 (1999).

[8] R. Combescot, Phys. Rev. Lett. 83, 3766 (1999).

[9] B. DeMarco and D. S. Jin, Science 285, 1703 (1999).

[10] K. M. O'Hara et al., Phys. Rev. Lett. 85, 2092 (2000).

[11] F. Schreck et al., Phys. Rev. A 64, 011402(R) (2001).

[12] A. G. Truscott et al., Science 291, 2570 (2001).

[13] F. Schreck et al., Phys. Rev. Lett. 87, 080403 (2001).

[14] V. Vuletic et al., Phys. Rev. Lett. 80, 1634 (1998).

[15] J. Fortagh, A. Grossmann, C. Zimmermann, and T. W. Hänsch, Phys. Rev. Lett. 81, 5310 (1998).

[16] J. Denschlag, D. Cassettari, and J. Schmiedmayer, Phys. Rev. Lett. 82, 2014 (1999).

[17] J. Reichel, W. Hänsel, and T. W. Hänsch, Phys. Rev. Lett. 83, 3398 (1999).

[18] H. Ott et al., Phys. Rev. Lett. 87, 230401 (2001).

[19] C. A. Regal, C. Ticknor, J. L. Bohn, and D. S. Jin, cond-mat/0209071

[20] H. L. Bethlem et al., Nature (London) 406, 491 (2000).

[21] V. J. Emery, Theory of the One-Dimensional Electron Gas, in Highly Conducting One-Dimensional Solids, edited by J. T. Devreese, R. P. Evard, and V. E. van Doren (Plenum, New York, 1979), p247.

[22] F. D. M. Haldane, J. Phys. C: Solid State Phys. 14, 2585 (1981).

[23] J. Voit, Rep. Prog. Phys. 58, 977 (1995).

[24] H. J. Schulz, Fermi Liquids and Non-Fermi Liquids, in Mesoscopic Quantum Physics, edited by E. Akkermans, G. Montambaux, J.-L. Pichard, and J. Zinn-Justin (Elsevier, Amsterdam, 1995), p533.

[25] W. Wonneberger, Phys. Rev. A 63, 063607 (2001).

[26] Gao Xianlong and W. Wonneberger, Phys. Rev. A 65, 033610 (2002).

[27] J. L. Cardy, J. Phys. A 17, L385 (1984). 
[28] S. Eggert and I. Affleck, Phys. Rev. B 46, 10866 (1992).

[29] M. Fabrizio and A. O. Gogolin, Phys. Rev. B 51, 17827 (1995).

[30] R. Egger and H. Grabert, Phys. Rev. Lett. 75, 3505 (1995).

[31] Y. Wang, J. Voit, and Fu-Cho Pu, Phys. Rev. B 54, 8491 (1996).

[32] A. E. Mattsson, S. Eggert, and H. Johannesson, Phys. Rev. B 56, 15615 (1997).

[33] J. Voit, Yupeng Wang, and M. Grioni, Phys. Rev. B 61, 7930 (2000).

[34] F. Gleisberg and W. Wonneberger, cond-mat/0208376

[35] D. C. Mattis and E. H. Lieb, J. Math. Phys. 6, 304 (1965).

[36] F. Lochmann, Diploma Thesis, University of Ulm, February 2001, unpublished.

[37] H. Q. Lin and J. E. Gubernatis, Computers in Physics 7, 400 (1993).

[38] The "proof" indicated in 25] to show that $S\left(N, \alpha_{1}\right)=N$ is flawed. It used an interchange of limiting procedures which is wrong, especially at strong coupling.

[39] K. Schönhammer and V. Meden, Am. J. Phys. 64, 1168 (1996).

[40] J. Friedel, Nuovo Cimento Suppl. 7, 287 (1958).

[41] F. Gleisberg, W. Wonneberger, U. Schlöder, and C. Zimmermann, Phys. Rev. A 62, 063602 (2000).

[42] M. Hillery, R. F. O'Connell, M. O. Scully, and E. P. Wigner, Phys. Rep. 106 (1984) 121.

[43] F. Gleisberg, W. P. Schleich, and W. Wonneberger, J. Phys. B: At. Mol. Opt. Phys. 34, 4645 (2001).

[44] W. P. Schleich, Quantum Optics in Phase Space, (Berlin, Wiley-VCH, 2001).

[45] P. Vignolo, A. Minguzzi, and M. P. Tosi, Phys. Rev. Lett. 85, 2850 (2000).

[46] A. Minguzzi, P. Vignolo, and M. P. Tosi, Phys. Rev. A 63, 063604 (2001).

[47] P. Vignolo, A. Minguzzi, and M. P. Tosi, Phys. Rev. A 64, 023421 (2001). 